\title{
Inflammatory Burden but Not Diabetes Mellitus Influences in Prognosis of Endovascular Revascularization in Peripheral Arterial Disease
}

\author{
Silvia Bleda, Joaquin de Haro, Cesar Varela, Ignacio Lopez de Maturana, \\ Javier Rodriguez, and Francisco Acin \\ Angiology and Vascular Surgery Department, Hospital Universitario de Getafe, 28905 Madrid, Spain \\ Correspondence should be addressed to Silvia Bleda; silbleik@yahoo.es
}

Received 30 November 2012; Accepted 9 January 2013

Academic Editors: F. Braet and J.-J. Li

Copyright (C) 2013 Silvia Bleda et al. This is an open access article distributed under the Creative Commons Attribution License, which permits unrestricted use, distribution, and reproduction in any medium, provided the original work is properly cited.

\begin{abstract}
Aims. To determine the effect of inflammatory preoperative burden on the incidence of reintervention and mortality after endovascular therapy (EVT) and to investigate if diabetes mellitus (DM) is involved in these results. Material and Methods. A prospective cohort study with 12-month follow-up period in patients with peripheral artery disease was carried out. hsCRP and fibrinogen assessments were determined. Results. 85 diabetic and 58 nondiabetic patients were included. An increase between basal hsCRP $(11.8 \mathrm{mg} / \mathrm{L}(10.2 ; 21.5)$ versus $4.3 \mathrm{mg} / \mathrm{L}(1.8 ; 13.9), P<0.001)$ and fibrinogen levels $(450 \mathrm{mg} / \mathrm{dL}(425 ; 479.1)$ versus $369 \mathrm{mg} / \mathrm{dL}$ (268; 419), $P<0.001)$ and the incidence of death during followup was found. A significant increase between higher hsCRP and fibrinogen basal levels and the incidence of reintervention during the follow-up period was also noted $(P=0.001$ and $P=0.04$, resp.,). There was no difference between DM and non-DM patients in the 1-year need of reintervention ( $33.3 \%$ versus $45 \%, P=0.15$, resp.,). Basal hsCRP and fibrinogen levels did not significantly differ between DM and non-DM patients who needed reintervention. Conclusions. The prognosis of the EVT is likely marked by the previous inflammatory load, regardless of DM.
\end{abstract}

\section{Introduction}

Peripheral arterial disease (PAD) is a growing health problem. It is a chronic and progressive disease affecting lower limbs arteria most commonly due to atherosclerosis. The role of inflammation in PAD is becoming increasingly recognized. Several inflammatory markers have been related to atherosclerosis, including high sensitive C-reactive protein (hsCRP) [1-4] and fibrinogen [4].

It has been proved that hsCRP acts by induction of cell adhesion molecule expression and mediation of lowdensity lipoprotein consumption by endothelial macrophages which can lead to atherosclerosis progression [5]. hsCRP has been associated with severity of PAD [3] and with a higher risk of cardiovascular events and long-term mortality in these patients $[6,7]$. Likewise, elevated fibrinogen levels are associated with greater risk for cardiovascular events and with all-cause mortality in PAD subjects $[4,8]$.
Endovascular therapy (EVT) is currently accepted as the first step treatment in a most of patients that suffer from PAD. Continuous advances in this technique have allowed to increasingly offer therapeutic treatment choice detrimental to the more aggressive intervention of open bypass. That first obtains a high rate of successful primary outcome [9-11]. However, the need of reintervention after EVT should not be taken lightly, being this close to $40 \%$ in the first 6 months after the procedure [12].

Diabetes mellitus (DM) is one of the most important risk factors for developing PAD [13]. Moreover, the prevalence of this disease is rapidly increasing in western world. Furthermore, the duration of the disease worsens its vascular prognosis [14]. The outcome of EVT in DM patients is not completely known. Controversial results about possible different results of EVT among diabetic and non-diabetic patients have been reported [15-17].

The goal of this study was to determine the effect of inflammatory preoperative burden on the incidence 
of reintervention and mortality, after EVT for PAD patients, and to investigate if the presence of DM affects these results.

\section{Material and Methods}

2.1. Study Design and Population. This prospective cohort study with a 12-month follow-up period examined the hypothetical relationship between inflammatory markers and prognosis of EVT as well as their association with DM in PAD patients.

Patients were considered as having PAD when their ankle-arm index was $<0.9$ or $>1.3$, when they presented symptoms of intermittent claudication, pain at rest, and/or trophic lesions.

Patients who underwent EVT for atherosclerotic obstructions lesions of iliac, femoral, popliteal, or distal arteries were screened. Patients with clinical evidence of infection including cellulitis, osteomyelitis, or deep space infection of the foot (Grade III, Category 6 Rutherford) were excluded. Other exclusion criteria were concurrent systemic infections, inflammatory or autoimmune disease, fever, immunosuppressive treatment, acute myocardial infarction, stroke, any major surgery or severe traumatism within 30 days prior to intervention, because of chronically increased hsCRP levels in these cases. Were also excluded those patients in which an open surgical bypass was indicated as first revascularization treatment option.

All patients included in this study provided their written informed consent. The study complied with the Declaration of Helsinki and was approved by the local ethics committee.

Cardiovascular risk factors, treatment, and general conditions from all patients were recorded the same day before the surgery.

DM was defined by a baseline blood glucose of $>126 \mathrm{mg} / \mathrm{dL}$, glycosilated haemoglobin Alc level greater than $0.06(>6.5 \%)$, or the need for glucose-lowering treatment accordingly WHO criteria. Hypertension was defined as having high blood pressure (systolic blood pressure $>140 \mathrm{~mm} \mathrm{Hg}$ and/or diastolic blood pressure $>90 \mathrm{~mm} \mathrm{Hg}$ ) and/or receiving antihypertensive treatment for at least 1 year before inclusion in the study. Dyslipidemia was defined as fasting total serum cholesterol level $>200 \mathrm{mg} / \mathrm{dL}$, LDL levels $\geq 140 \mathrm{mg} / \mathrm{dL}$, triglycerides levels $\geq 150 \mathrm{mg} / \mathrm{dL}$, HDL levels $<40 \mathrm{mg} / \mathrm{dL}$, or antidyslipidemic treatment. Smoking habit was defined as active smoker when the patient smoked at the time of the inclusion or gave up the habit in a period lower than 6 months.

\subsection{Blood Collection and Assays for Inflammatory Markers.} Two markers were used to determine the systemic inflammatory burden, hsCRP, and fibrinogen. Antecubital venous blood samples for determination of hsCRP and fibrinogen were collected at preintervention ward before EVT. Samples were spun at $3000 \mathrm{rpm}$ for 20 minutes and then frozen at $-70^{\circ} \mathrm{C}$ until its analysis. Levels of hsCRP were measured by ultrasensitive automated immunoassay (Roche Diagnostics), with detection in the lower limit of $0.2 \mathrm{mg} / \mathrm{L}$ and a variation rate of $4.2 \%$ in $4 \mathrm{mg} / \mathrm{L}$ and $6.3 \%$ in $1 \mathrm{mg} / \mathrm{L}$ [18].
2.3. End Points and Study Protocol. Reintervention events included any new procedure of balloon angioplasty/stenting or open revascularization with a bypass vein/graft, as well as any major amputation during the follow-up period. All patients were clinically monitored for postoperative initial complications reporting prior to discharge. Patients were routinely followed up for 1 year in the outpatient clinic by a vascular surgeon blinded for basal inflammatory burden of the patients to analyze the occurrence of reinterventions and/or death. Ankle-braquial index (ABI) measurement, evaluation of patient complaints, and physical reexamination were performed at all visits in all patients. Per protocol, all patients received duplex ultrasound imaging surveillance at $1,3,6$, and 12 months, which is consistent with our standard practice. The duplex ultrasound examination was performed in an accredited vascular laboratory by an experimental sonographer. An arteriography was carried out during the followup in those patients who suffered a worsening in their symptoms with an increase in one category in the Rutherford scale, decrease in $\mathrm{ABI}>0.2$ from the maximum postprocedural level, clinical worsening of tissue loss or the appearance control duplex-scan of a restenosis area, defined as an increase in peak systolic ratio $\geq 2.4$ [19]. Angiographic restenosis was classified as $50 \%$ or greater diameter reduction at the dilated vessel segment.

2.4. Statistical Methods. Statistical analysis was performed using SPSS, version 15.0 for Windows (SPSS, Chicago, IL, USA). The distribution of all inflammatory markers was nonGaussian (rightward skewed) and is presented as median and interquartile range. To analyze the association between inflammatory markers and mortality and reinterventions events, Mann-Whitney $U$ test was used. Likewise, this test was performed in order to find statistical differences in basal inflammatory markers in diabetic and nondiabetic groups and the possible association of these with mortality and reintervention events. A difference with a $P$ value less than 0.05 was considered statistically significant.

The sample size needed to obtain significant differences regarding the requirement of reintervention, with $80 \%$ degree of statistical power and a type error alpha of 0.05 , was calculated on the basis of prior studies analyzing plasma hsCRP and fibrinogen levels $[6,12]$ and was 50 patients.

\section{Results and Discussion}

3.1. Results. 317 patients undergoing revascularization surgery treatment were screened. 87 patients were excluded because they underwent bypass surgery. 72 patients who underwent EVT during inclusion period were excluded for suffering Rutherford category 6. Fifteen patients were excluded by other exclusion criteria (stated at Section 2). 143 patients (143 lower limbs) were finally accepted for analysis. There were 85 diabetic patients $(59.5 \%)$ and 58 nondiabetic patients (40.5\%). Subject demographics and risk factor features are presented in Table 1.

The indication for the revascularization was critical limb ischemia, defined as rest pain or tissue loss (Rutherford 
TABLE 1

\begin{tabular}{lc}
\hline Features & $N(\%)$ \\
\hline Age (average \pm DS) & $70.8 \pm 8.1$ \\
Male & $102(79.7)$ \\
Hypertension & $90(70.3)$ \\
Diabetes mellitus & $82(64.1)$ \\
Dyslipidemia & $48(37.5)$ \\
Prior acute myocardial infarction & $44(34.4)$ \\
Prior stroke & $14(10.9)$ \\
Chronic renal disease & $24(18.8)$ \\
Current smoking & $50(39.1)$ \\
Ex-smokers & $24(18.8)$ \\
\hline
\end{tabular}

category 4 and 5), in 120 (84\%) subjects and intermittent claudication (Rutherford category 3) in 23 (16\%).

Distribution of revascularized lesion areas included the iliac artery in 23 patients (16.1\%), superficial femoral artery in $66(46.2 \%)$, popliteal artery in $8(5.6 \%)$, arteries below knee in $16(11.2 \%)$, and multilevel treatment in 30 (20.9\%).

EVT was performed with an inguinal approach in all cases. Before angioplasty/stenting, patients received heparin intravenously. Primary stenting was performed in iliac area [10]. Lesions in infrainguinal area were treated by means of balloon angioplasty. In the infrainguinal group, $13 \%$ of the patients crossed over to secondary stenting, mainly because of suboptimal PTA result defined as flow-limiting dissection or residual stenosis of $>30 \%$. Immediate technical success was defined as residual stenosis $<30 \%$.

All patients maintained treatment with AAS $100 \mathrm{mg} / \mathrm{d}$ and statins after surgery, being implemented in those who were not treated.

3.2. Influence of Diabetes Mellitus in the Relationship between Inflammatory Markers and Long-Term Reinterventions. The analysis of the need of reintervention at 1 year, after excluding the 10 patients who had exitus during followup, was carried out in 133 patients, of which $81(60.9 \%)$ were diabetic patients and $52(39.1 \%)$ nondiabetic patients. There were $48(36 \%)$ reinterventions at the end of the first year of followup.

The median of basal inflammatory markers in the group of nonreintervention patients was $3.3 \mathrm{mg} / \mathrm{L}$ [1.1; 8.7] for hsCRP and $343 \mathrm{mg} / \mathrm{dL}$ [235; 382.5] for fibrinogen. Among patients who needed some kind of reintervention along the year of followup, it was obtained a median of $8.5 \mathrm{mg} / \mathrm{L}$ [4; 15.4] for hsCRP and $368.3 \mathrm{mg} / \mathrm{dL}$ [284.5; 430] for fibrinogen. Basal hsCRP and fibrinogen levels were significantly higher in reintervention patients $(P=0.001$ and $P=0.04$, resp., $)$ (Figures 1 and 2).

The need of reintervention was stratified according to clinical presence of DM. There was no statistically significant difference between DM and non-DM patients in the 1-year need of reintervention ( $33.3 \%$ versus $40.3 \%, P=0.15$, resp.,).

When basal hsCRP and fibrinogen levels were analyzed according to clinical presence of DM, there were no differences among both groups (4.6 [1.4-12] hsCRP in $\mathrm{DM}$ versus 4.8 [3.4-9] hsCRP in non-DM, $P=0.37$;
350 [281.8-407.1] fibrinogen in DM versus 348.3 [276.8-450] fibrinogen in non-DM, $P=0.97$ ). (Figures 1 and 2). In diabetes patients, the median of hsCRP in the subgroup of nonreintervention (66.6\%) was significantly lower than in the reintervention subgroup (33.3\%) $(3.6 \mathrm{mg} / \mathrm{L}$ [1.2-9.5] versus $12 \mathrm{mg} / \mathrm{L}$ [1.8-18.5], $P=0.014$, resp.,). However, no differences were observed when fibrinogen levels were analyzed [350 mg/dL [278.9-389.2] in nonreintervention group versus 338.9 [282.5-430] in reintervention group, $P=0.26$ ] (Figure 3).

Among non-DM patients, greater basal hsPCR levels were found in the reintervention group (40.4\%) than in nonreintervention group [9.7 $\mathrm{mg} / \mathrm{L}$ [4.7-16.9] versus $3.9 \mathrm{mg} / \mathrm{L}$ [2.7-5.9], resp., $P<0.001]$, as well as basal fibrinogen levels [424.5 mg/dL [347.5-472.5] versus $297.6 \mathrm{mg} / \mathrm{dL}$ [240343], resp., $P=0.001$ ] (Figure 4 ).

\subsection{Influence of DM in the Relationship between Inflammatory} Markers and Mortality. Exitus rate at one year of followup was 7\% (10 patients). Higher baseline levels of hsCRP $(18.8 \mathrm{mg} / \mathrm{L}$ [7.8; 25.5] versus $3.7 \mathrm{mg} / \mathrm{L}$ [1.8; 9.4], $P<0.001)$ and fibrinogen $(450 \mathrm{mg} / \mathrm{dL}$ [425; 479.1] versus $340 \mathrm{mg} / \mathrm{dL}$ [265; 403.2], $P<0.001$ ) were significantly found among patients that suffered exitus during followup.

The rate of exitus was similar in diabetic patients compared with nondiabetic patients ( 4 versus 6 patients, $P=$ 0.09 , resp.). In diabetes patients, the median of hsCRP in the subgroup of non-exitus was significantly lower than in the exitus subgroup $(5.4 \mathrm{mg} / \mathrm{L}(1.6-12)$ versus $21.7 \mathrm{mg} / \mathrm{L}$ (18.525), $P<0.001$, resp.), as well as basal fibrinogen levels (350/dL (280-406.5) versus $424 \mathrm{mg} / \mathrm{dL}$ (372-475), resp., $P=$ $0.04)$.

Among non-DM patients, greater basal hsPCR levels were found in patients that suffered exitus during follow-up than nonexitus patients $(10.9 \mathrm{mg} / \mathrm{L}(9.5-129)$ versus $4.6 \mathrm{mg} / \mathrm{L}$ (3.4-8.99), resp., $P=0.001)$, as well as basal fibrinogen levels (450 mg/dL (442.7-448.5) versus $328 \mathrm{mg} / \mathrm{dL}$ (245-406.3), resp., $P=0.006)$.

\section{Discussion}

PAD is a relatively common condition affecting up to $20 \%$ of the population older than 65 years and is becoming a growing health problem $[20,21]$. It is associated with a high cardiovascular morbidity and mortality [22] and in its symptomatic form represents an important cause of disability [23].

Because EVT is minimally invasive, unless a major bleeding complication occurs, it has become an accepted therapeutic alternative to open surgery in patients with PAD. Initial technical success rates for EVT are well accepted [10, $24,25]$. However, the percentage of reinterventions that these patients will undergo is nearly $40 \%$ in the first 6 months after the procedure. The accurate causes of this early failure are not well understood. A previous study has shown that the inflammatory burden is able to predict the prognosis of these interventions regarding long-term reintervention needing and mortality in patients with PAD [26]. 

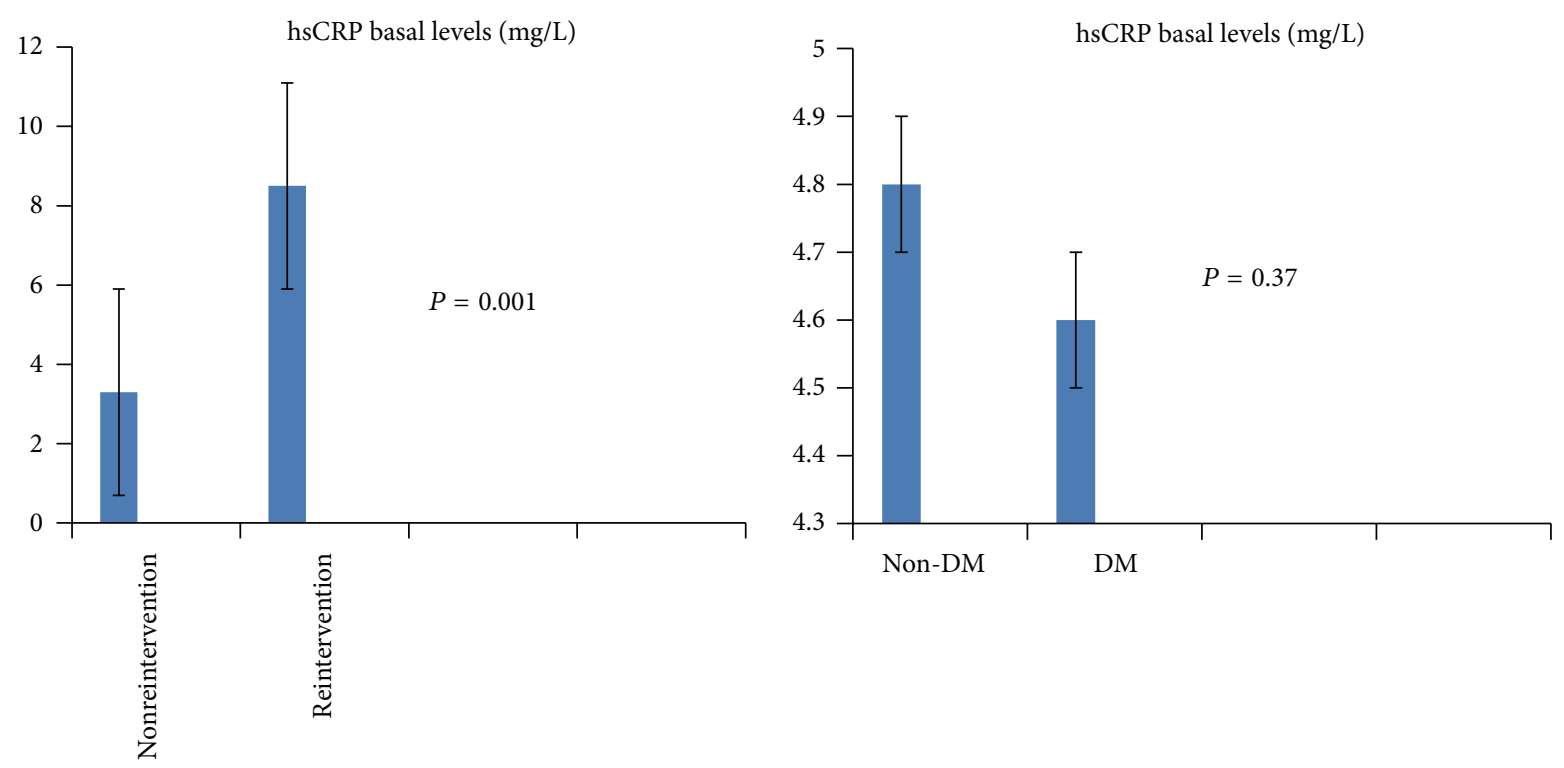

FIGURE 1
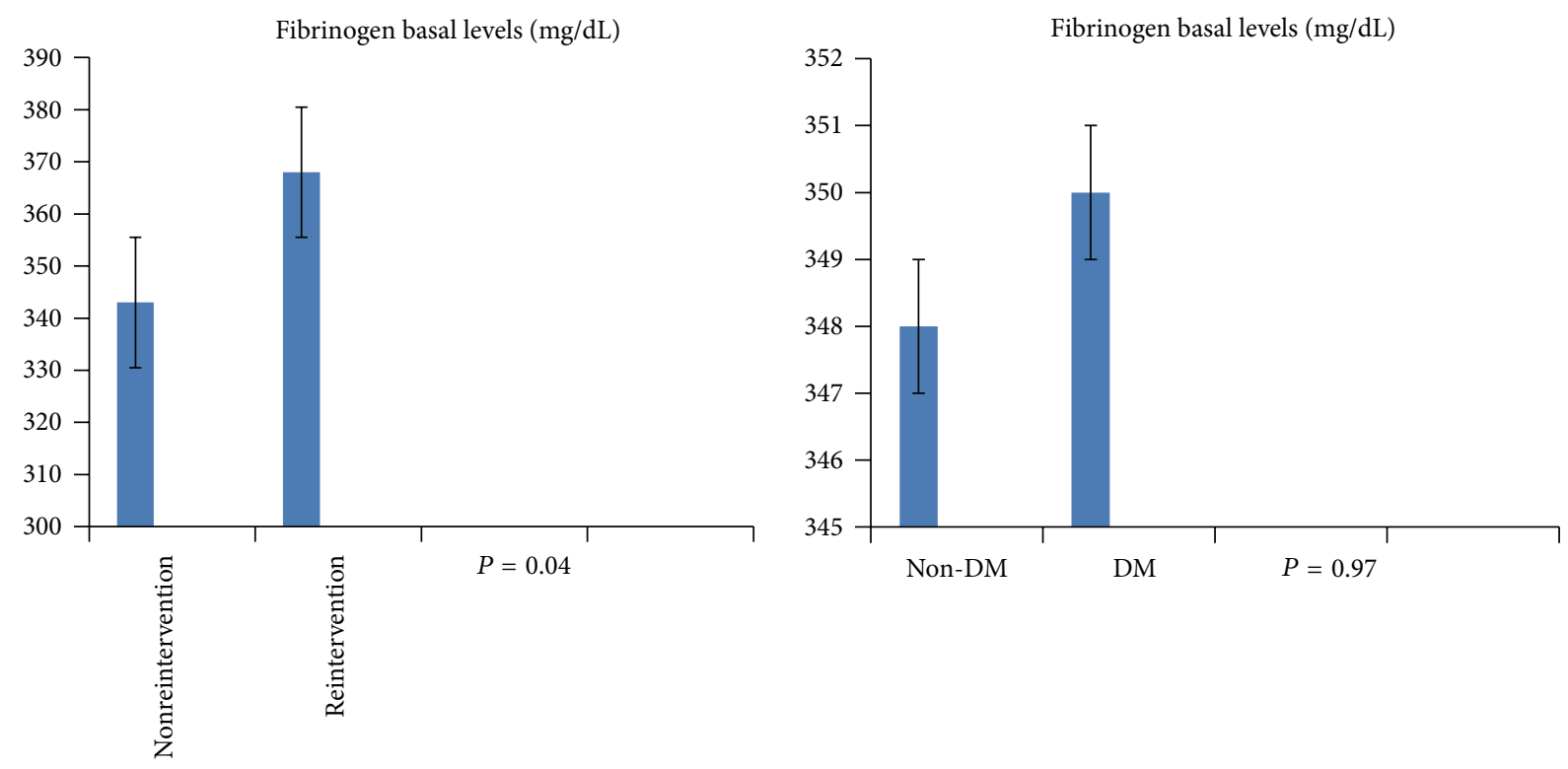

Figure 2

We hypothesized that DM along with the basal inflammatory burden are cofactors that confer greater risk for exitus and EVT failure for PAD patients. Although the mechanisms of diabetic vascular disease remain incompletely understood, considerable data suggest that hyperglycemia itself causes vascular damage. Furthermore, the outcome of EVT in DM patients is not yet well known. Some authors have found no difference in outcomes for DM patients undergoing EVT $[15,16]$, whereas others have found worse results in these patients [17]. However, DM could be an increasingly important independent factor because of the rising number of PAD patients with DM.

Meanwhile, inflammatory markers from asymptomatic patients have been showed to be predictor risk factors for the development of subsequent PAD [27]. Moreover, hsCRP levels before and after intervention have been associated with 6-month restenosis rates after femoropopliteal angioplasty, finding a direct relationship between the inflammation triggered by the EVT itself and the risk of restenosis $[12,28]$.

Nevertheless, the possible mechanism of interaction between DM and inflammation in PAD patients is disputed. DM may accelerate the progression of atherosclerosis or act through other separate pathways.

To test our hypothesis, a prospective cohort study in PAD patients underwent to EVT with a 12-month followup was conducted. Its specific aim was to analyze the association of $\mathrm{DM}$ and inflammatory markers among them and with the rate of exitus and reintervention after EVT. 
Diabetes mellitus subgroup
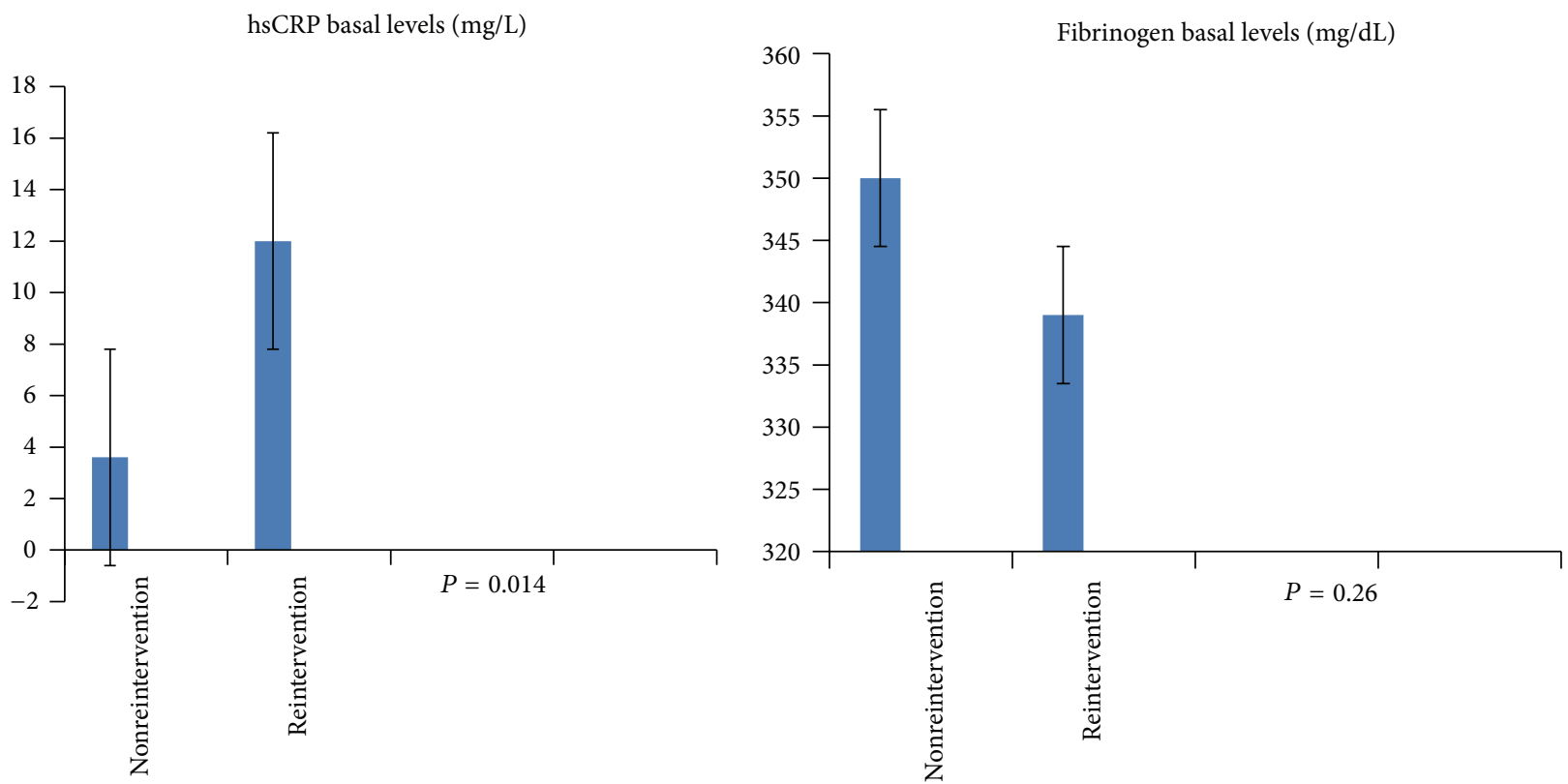

Figure 3

Non-diabetes mellitus subgroup
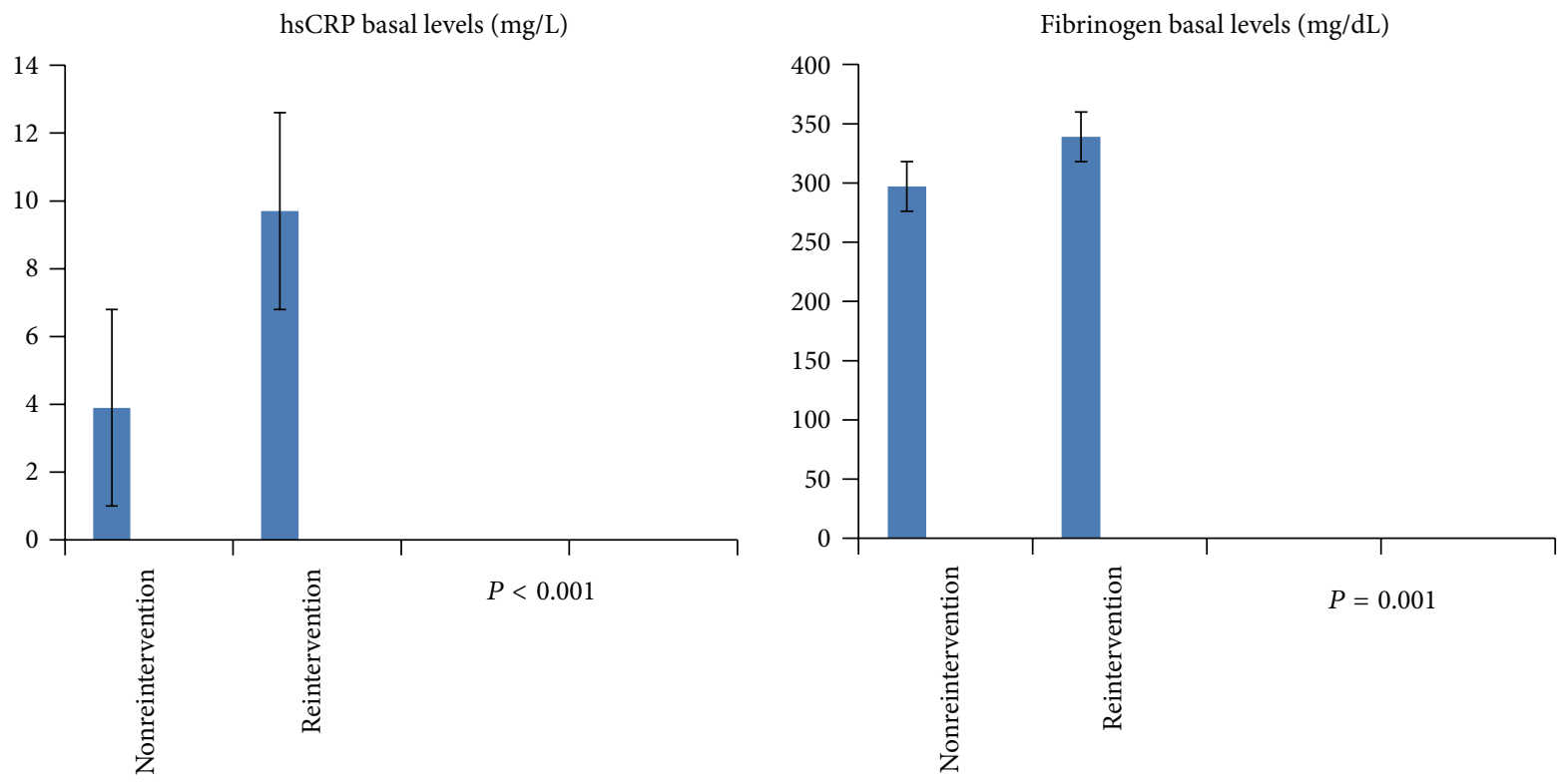

FIGURE 4

Our data show that preintervention hsCRP and fibrinogen levels were significantly associated with the occurrence of reintervention and exitus at 1 year of followup. Further, higher baseline inflammatory levels indicate an increased risk for reintervention as a new EVT, bypass and/or amputation of the limb as well as a greater risk for exitus.

Nonetheless, no independent association among DM and EVT outcomes or exitus was found in these patients. The rate of reinterventions and exitus was similar in diabetic and nondiabetic patients. Moreover, no differences were found in basal hsPCR and fibrinogen levels according to presence of DM. Such that, the basal inflammatory burden in DM patients was like in non-DM patients.

The current study shows that baseline levels of hsPCR and fibrinogen are independent risk factors of a worse prognosis of EVT. Likewise, although EVT per se, is able to produce an inflammatory process on the arterial wall, the present study proves that the effect of the systemic inflammatory status on 
the results of EVT could be independent of that effect upon the vascular wall.

Data found about the relationship between greater basal levels of inflammatory markers and a poor life expectative are consistent to others investigations $[4,6]$. In this prospective evaluation of patients undergoing EVT, our results show that higher plasma circulating levels of systemic inflammation markers (hsCRP and fibrinogen) are associated with increased mortality during followup.

But although, DM may be involved in the outcomes of EVT, it has not been found that DM itself was an independent predictor of increased rate of reintervention or exitus.

All previous data suggest that the prognosis of EVT is marked by the previous inflammatory load but not by DM.

Therefore, systemic inflammation may be involved in the etiopathogenesis of arterial restenosis. Thus inflammation is related to endothelial dysfunction in PAD patients [29]. The ability of hsCRP to reduce nitric oxide synthase expression supports this effect [30]. Biologically is plausible to hypothesize that the inflammatory process triggers vascular smooth muscle cell proliferation and constrictive neointima formation being involved in the pathogenesis of postangioplasty restenosis [12].

The inflammatory process plays a pivotal role in the pathogenesis and evolution of atherosclerosis as well as the development of its clinical manifestations as PAD. Whether circulating hsCRP serves as just a marker or plays a direct biologic role in lesion development remains an area of active investigation.

Moreover, DM patients are a heterogeneous group, and further investigation is required to more completely understand the impact of DM on PAD and on the outcomes of EVT.

\section{Conclusions}

The impact of DM on patency of EVT in PAD patients is probably less incidental than the previous inflammatory load that these patients present. The prognosis of the EVT is likely marked by the previous inflammatory load but not by DM.

\section{References}

[1] P. M. Ridker, M. Cushman, M. J. Stampfer, R. P. Tracy, and C. H. Hennekens, "Plasma concentration of C-reactive protein and risk of developing peripheral vascular disease," Circulation, vol. 97, no. 5, pp. 425-428, 1998.

[2] I. Tzoulaki, G. D. Murray, A. J. Lee, A. Rumley, G. D. O. Lowe, and F. G. R. Fowkes, "C-reactive protein, interleukin6, and soluble adhesion molecules as predictors of progressive peripheral atherosclerosis in the general population: Edinburgh Artery Study," Circulation, vol. 112, no. 7, pp. 976-983, 2005.

[3] J. De Haro, F. Acín, F. J. Medina et al., "Relationship between the plasma concentration of C-reactive protein and severity of peripheral arterial disease," Clinical Medicine, no. 3, pp. 1-7, 2008.

[4] J. E. van Loon, M. P. de Maat, J. W. Deckers, R. T. van Domburg, and F. W. Leebeek, "Prognostic markers in young patients with premature coronary heart disease," Atherosclerosis, vol. 224, no. 1, pp. 213-217, 2012.
[5] T. P. Zwaka, V. Hombach, and J. Torzewski, "C-reactive proteinmediated low density lipoprotein uptake by macrophages: implications for atherosclerosis," Circulation, vol. 103, no. 9, pp. 1194-1197, 2001.

[6] C. D. Owens, P. M. Ridker, M. Belkin et al., "Elevated Creactive protein levels are associated with postoperative events in patients undergoing lower extremity vein bypass surgery," Journal of Vascular Surgery, vol. 45, no. 1, pp. 2-9, 2007.

[7] E. Rossi, L. M. Biasucci, F. Citterio et al., "Risk of myocardial infarction and angina in patients with severe peripheral vascular disease predictive role of C-reactive protein," Circulation, vol. 105, no. 7, pp. 800-803, 2002.

[8] W. S. Speidl, A. Zeiner, M. Nikfardjam et al., "An increase of C-reactive protein is associated with enhanced activation of endogenous fibrinolysis at baseline but an impaired endothelial fibrinolytic response after venous occlusion," Journal of the American College of Cardiology, vol. 45, no. 1, pp. 30-34, 2005.

[9] I. L. Gordon, R. M. Conroy, M. Arefi, J. M. Tobis, E. A. Stemmer, and S. E. Wilson, "Three-year outcome of endovascular treatment of superficial femoral artery occlusion," Archives of Surgery, vol. 136, no. 2, pp. 221-228, 2001.

[10] L. Norgren, W. R. Hiatt, J. A. Dormandy, M. R. Nehler, K. A. Harris, and F. G. R. Fowkes, "Inter-society consensus for the management of peripheral arterial disease (TASC II)," Journal of Vascular Surgery, vol. 45, no. 1, pp. S5-S67, 2007.

[11] F. Pozzi Mucelli, M. Fisicaro, L. Calderan et al., "Percutaneous revascularization of femoropopliteal artery disease: PTA and PTA plus stent. Results after six years' follow-up," Radiologia Medica, vol. 105, no. 4, pp. 339-349, 2003.

[12] M. Schillinger, M. Exner, W. Mlekusch et al., "Endovascular revascularization below the knee: 6-Month results and predictive value of C-reactive protein level," Radiology, vol. 227, no. 2, pp. 419-425, 2003.

[13] M. H. Criqui, "Peripheral arterial disease-epidemiological aspects," Vascular Medicine, vol. 6, supplement 3, pp. 3-7, 2001.

[14] W. K. Al-Delaimy, A. T. Merchant, E. B. Rimm, W. C. Willett, M. J. Stampfer, and F. B. Hu, "Effect of type 2 diabetes and its duration on the risk of peripheral arterial disease among men," American Journal of Medicine, vol. 116, no. 4, pp. 236-240, 2004.

[15] S. M. Surowiec, M. G. Davies, S. W. Eberly et al., "Percutaneous angioplasty and stenting of the superficial femoral artery," Journal of Vascular Surgery, vol. 41, no. 2, pp. 269-278, 2005.

[16] M. J. Costanza, L. A. Queral, M. P. Lilly et al., "Hemodynamic outcome of endovascular therapy for TransAtlantic InterSociety Consensus type B femoropopliteal arterial occlusive lesions," Journal of Vascular Surgery, vol. 39, no. 2, pp. 343-350, 2004.

[17] C. J. Abularrage, M. F. Conrad, L. A. Hackney et al., "Longterm outcomes of diabetic patients undergoing endovascular infrainguinal interventions," Journal of Vascular Surgery, vol. 52, no. 2, pp. 314-322, 2010.

[18] S. Eda, J. Kaufmann, W. Roos et al., "Development of a new microparticle-enhanced turbidimetric assay for C-reactive protein with superior features in analytical sensitivity and dynamic range," Journal of Clinical Laboratory Analysis, vol. 12, pp. 137144, 1998.

[19] C. Ranke, A. Creutzig, and K. Alexander, "Duplex scanning of the peripheral arteries: correlation of the peak velocity ratio with angiographic diameter reduction," Ultrasound in Medicine and Biology, vol. 18, no. 5, pp. 433-440, 1992.

[20] W. T. Meijer, A. W. Hoes, D. Rutgers, M. L. Bots, A. Hofman, and D. E. Grobbee, "Peripheral arterial disease in the elderly: 
The Rotterdam Study," Arteriosclerosis, Thrombosis, and Vascular Biology, vol. 18, no. 2, pp. 185-192, 1998.

[21] J. M. Murabito, J. C. Evans, K. Nieto, M. G. Larson, D. Levy, and P. W. F. Wilson, "Prevalence and clinical correlates of peripheral arterial disease in the Framingham Offspring Study," American Heart Journal, vol. 143, no. 6, pp. 961-965, 2002.

[22] M. H. Criqui, R. D. Langer, A. Fronek et al., "Mortality over a period of 10 years in patients with peripheral arterial disease," The New England Journal of Medicine, vol. 326, no. 6, pp. 381386, 1992.

[23] G. Barletta, S. Perna, C. Sabba, A. Catalano, C. O’Boyle, and G. Brevetti, "Quality of life in patients with intermittent claudication: relationship with laboratory exercise performance," Vascular Medicine, vol. 1, no. 1, pp. 3-7, 1996.

[24] M. Lenti, E. Cieri, P. De Rango et al., "Endovascular treatment of long lesions of the superficial femoral artery: results from a multicenter registry of a spiral, covered polytetrafluoroethylene stent," Journal of Vascular Surgery, vol. 45, no. 1, pp. 32-39, 2007.

[25] T. R. Vogel, L. E. Shindelman, G. B. Nackman et al., "Efficacious use of nitinol stents in the femoral and popliteal arteries," Journal of Vascular Surgery, vol. 38, no. 6, pp. 1178-1184, 2003.

[26] S. Bleda, J. de Haro, F. Acin, C. Varela, L. Esparza, and I. Lopez de Maturana, "The inflammatory burden predicts long term outcomes in endovascular therapy in peripheral arterial disease," Annals of Vascular Surgery. In press.

[27] G. Engström, D. Site-Flondell, B. Lindblad, L. Janzon, and F. Lindgärde, "Risk of treatment of peripheral arterial disease is related to inflammation-sensitive plasma proteins: A Prospective Cohort Study," Journal of Vascular Surgery, vol. 40, no. 6, pp. 1101-1105, 2004.

[28] M. Schillinger, M. Haumer, G. Schlerka et al., "Restenosis after percutaneous transluminal angioplasty in patients with peripheral artery disease: the role of inflammation," Journal of Endovascular Therapy, vol. 8, pp. 477-483, 2001.

[29] J. De Haro Miralles, E. Martínez-Aguilar, A. Florez, C. Varela, S. Bleda, and F. Acin, "Nitric oxide: Link between endothelial dysfunction and inflammation in patients with peripheral arterial disease of the lower limbs," Interactive Cardiovascular and Thoracic Surgery, vol. 9, no. 1, pp. 107-112, 2009.

[30] S. Verma, M. A. Kuliszewski, S. H. Li et al., "C-reactive protein attenuates endothelial progenitor cell survival, differentiation, and function: further evidence of a mechanistic link between C-reactive protein and cardiovascular disease," Circulation, vol. 109, no. 17, pp. 2058-2067, 2004. 


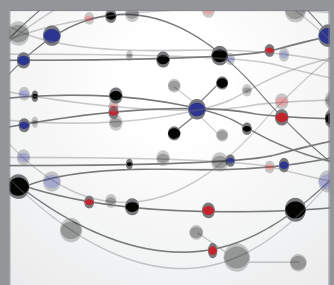

The Scientific World Journal
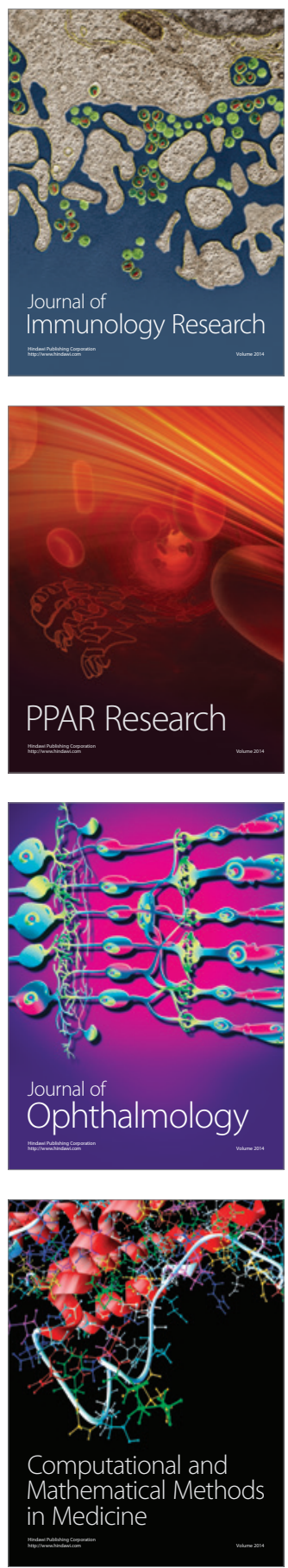

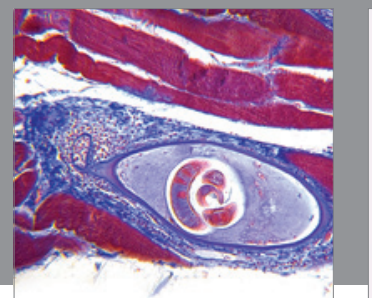

Gastroenterology

Research and Practice
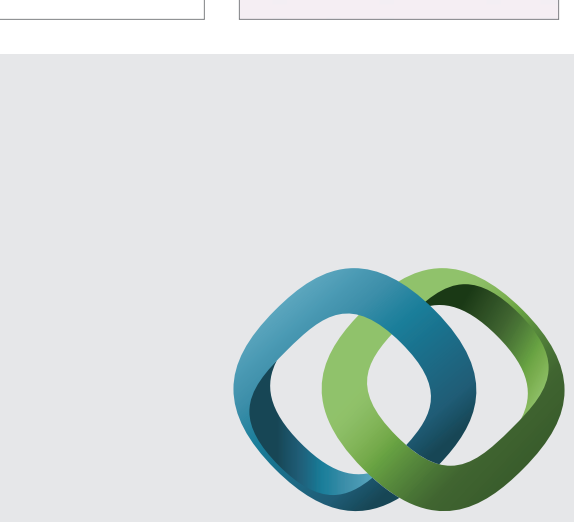

\section{Hindawi}

Submit your manuscripts at

http://www.hindawi.com
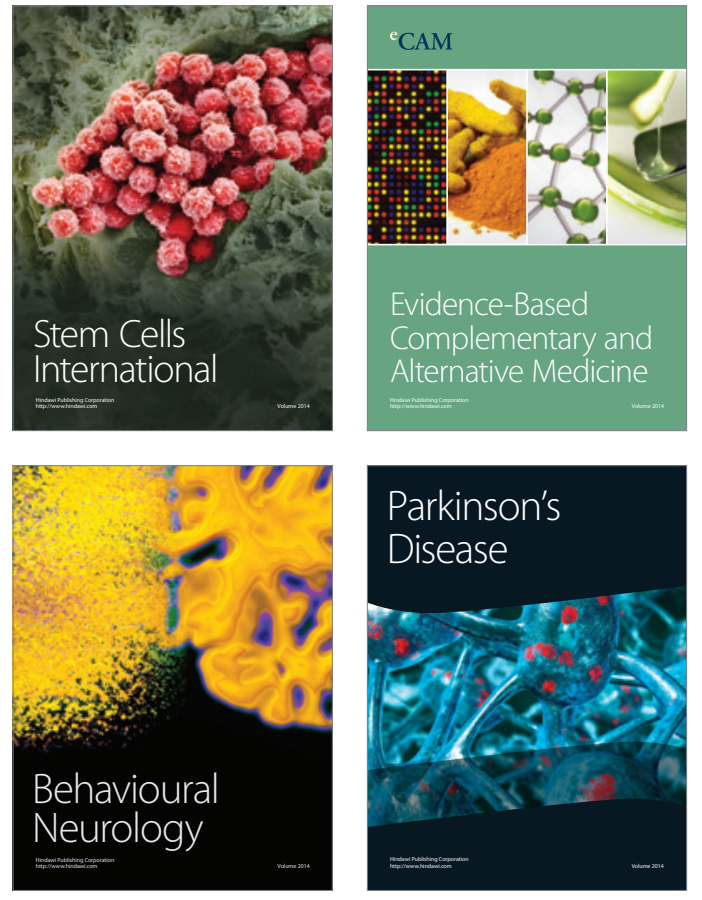
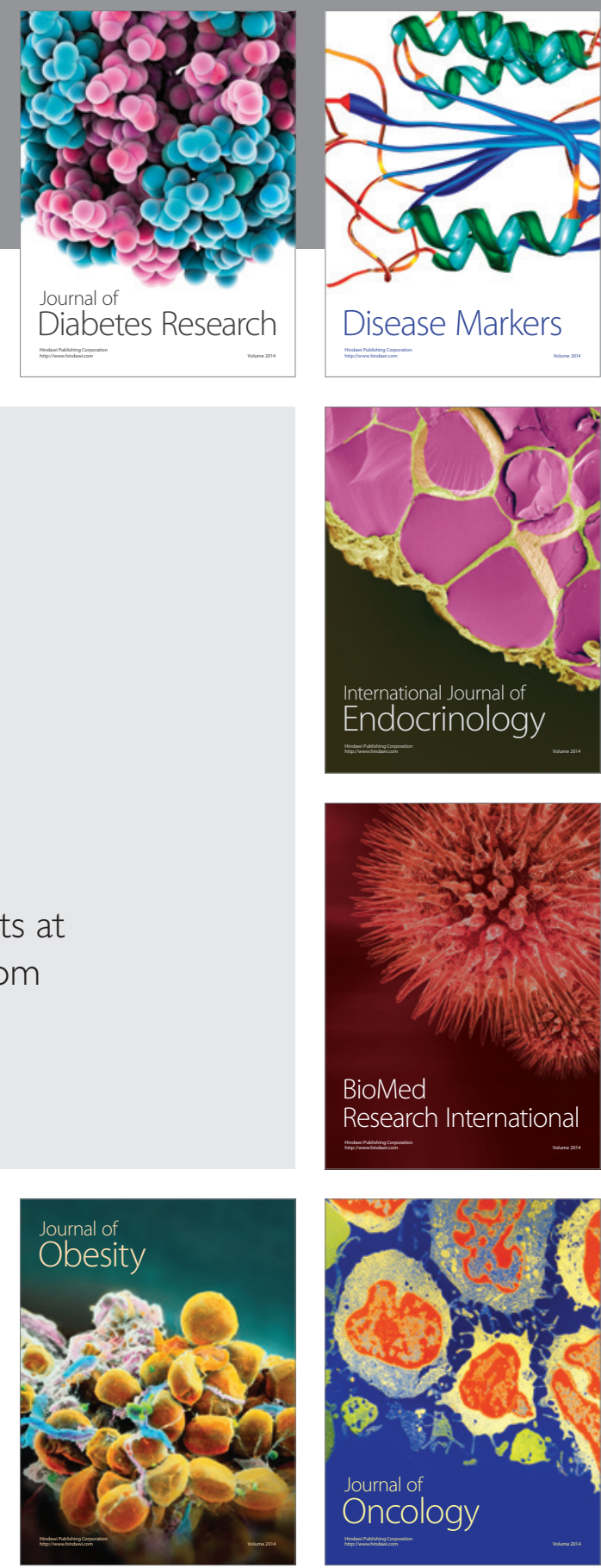

Disease Markers
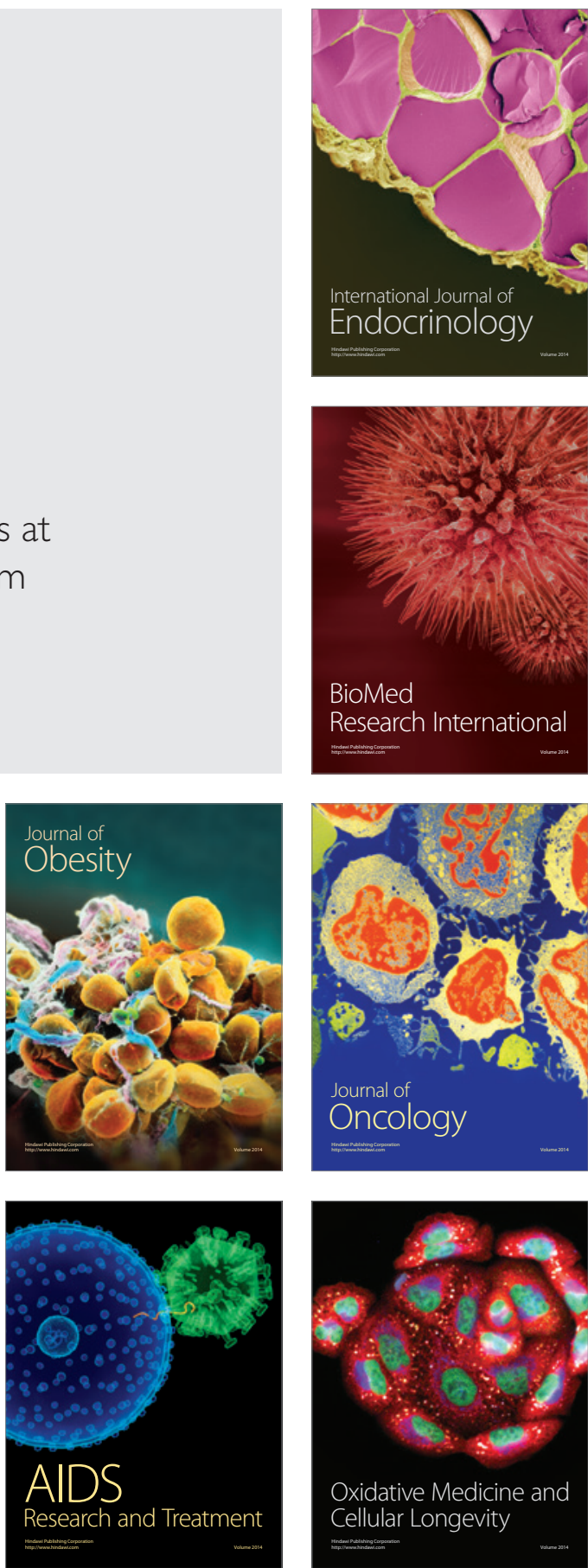